
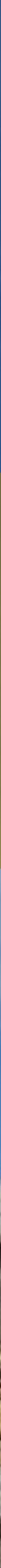


\section{Panta Rei \\ Revista Digital de Ciencia \\ y Didáctica de la Historia}

\section{6}

Revista anual

Fecha de inicio: 1995

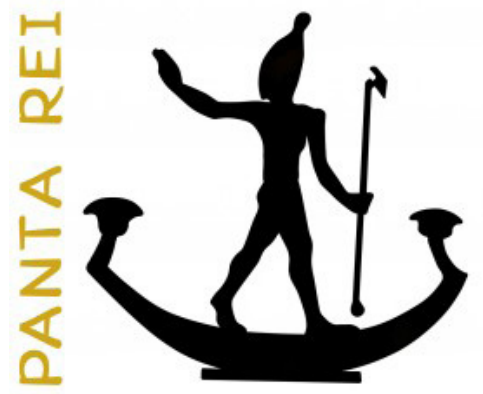

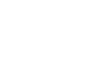




\section{CONSEJO DE REDACCIÓN}

\section{Coordinador editorial}

Egea Vivancos, Alejandro

[Didáctica de las Ciencias Sociales, UMU]

\section{Editores}

Botí Hernández, Juan Jesús

[CEPOAT, UMU]

Meseguer Gil, Antonio José

[CEPOAT, UNED]

Sáez Giménez, David Omar

[CEPOAT, UMU]

Sánchez Mondéjar, Celso Miguel

[CEPOAT, UMU]

\section{Secretaria}

Arias Ferrer, Laura

[Didáctica de las Ciencias Sociales, UMU]

\section{Responsable informático}

Martínez García, José Javier

[CEPOAT, UMU]

\section{Traducción y corrección lingüística}

Martínez Martínez, Cristina

[Sociedad Española de Lenguas Modernas]

Albaladejo Albaladejo, Sara

[ISEN, UMU]

\section{CONSEJO ASESOR}

Albero Muñoz, M. ${ }^{a}$ del Mar

[H. ${ }^{a}$ del Arte, UMU]

Chapman, Arthur

[History Education, UCL, Reino Unido]

Cobacho López, Ángel

[Derecho, UMU]

Egea Bruno, Pedro M. ${ }^{\text {a }}$

[Historia Contemporánea, UMU]

García Atienzar, Gabriel

[Prehistoria, UA]

González Monfort, Neus

[Didáctica de las Ciencias Sociales, UAB]

Haber Uriarte, María

[Prehistoria, UMU]

Hutson, Scott R.

[Anthropology, UK, EEUU]

Irigoyen López, Antonio

[Historia Moderna, UMU]

Mahony, Simon

[Digital Humanities, UCL, Reino Unido]

Marsilla de Pascual, Francisco Reyes

[Técnicas historiográficas, UMU]

Miralles Maldonado, José Carlos

[Filología Clásica, UMU]

Molina Gómez, José Antonio

[Historia Antigua, UMU]

Noguera Celdrán, José Miguel

[Arqueología, UMU]

Pérez Molina, Miguel Emilio

[Filología Clásica, UMU]

Prados Martínez, Fernando

[Arqueología, UA]

Sánchez Ibáñez, Raquel

[Didáctica de las Ciencias Sociales, UMU]

Sancho Gómez, Miguel Pablo

[Educación, UCAM]

Vilar García, María José

[Historia Contemporánea, UMU]

Zamora López, José Ángel

[Próximo Oriente Antiguo, CCHS-CSIC] 

Artículos

Los orígenes de la tecnología a debate: una revisión de las primeras industrias líticas.

Arturo Cueva Temprana.

De arqueología menorquina: Maria Lluïsa Serra Belabre y los círculos talayóticos de Sant Vicenç d' Alcaidús (Alaior, Menorca).

Octavio Torres Gomariz.

Nuevas cuestiones sobre el anfiteatro de Zaragoza.

José David Mendoza Álvarez.

Castidad o castigo. El estupro de las Vestales como símbolo de desorden social en Roma.

Juan Antonio Montalbán Carmona.

La Historia antigua en la Biblioteca de Focio.

Juan Luis Posadas Sánchez.

Usos sociales de la historia. La estrategia de Olga Cossettini, Rosario, 1935-1943.

Paula Caldo, Micaela Pellegrini Malpiedi y Agustina Mosso

Contribuciones a la didáctica de la Historia a través del método de análisis del objeto: como ejemplo... una "vasulla".

Nayra Llonch Molina y Verónica Parisi Moreno.

How are digital methods changing research in the study of the classical world? An EpiDoc case study. Katherine Steiner y Simon Mahony.

\section{Reseñas}

I Congreso Internacional "Creando ciudadanos, construyendo identidades. El uso del patrimonio material e inmaterial en la enseñanza de la historia."

José Díaz Serrano, Ainoa Escribano Miralles, Ana Isabel Ponce Gea y David Verdú González 151

Beckert, S. (2014). Empire of cotton: A global history. New York: Alfred A Knopf. 640 págs.

Ricky D. Mullins Jr..

Coumert, M. y Dumézil, B. (2013): Los reinos bárbaros en Occidente (traducción de Peinado Santaella, R. G.: Les royaumes barbares en Occident, Presses Universitaires de France, 2010). Editorial Universidad de Granada. Granada. 156 págs.

José Ángel Castillo Lozano.

La prehistoria en Las tres edades de Buster Keaton.

Alberto Lombo Montañés y Esther Rodríguez Ortiz.

Normas de publicación/Publishing rules 



\title{
Contribuciones a la didáctica de la Historia a través del método de análisis del objeto: como ejemplo... una "vasulla"
}

\author{
Contributions to the teaching and learning of History through objects \\ analysis: as an example... a "vasulla"
}

Nayra Llonch Molina ${ }^{1}$

Universidad de Lleida

Verónica Parisi Moreno²

Universidad de Lleida

Recibido: $18 / 06 / 2016$

Aceptado: 20/07/2016

Para citar este artículo: Llonch Molina, N. y Parisi Moreno, V. (2016). Contribuciones a la didáctica de la Historia a través del método de análisis del objeto: como ejemplo... una "vasulla". Panta Rei. Revista Digital de Ciencia y Didáctica de la Historia, 111-124.

ISSNe: 2386-8864

DOI: $10.6018 /$ pantarei/2016/7

\section{Resumen}

El propósito de este trabajo de investigación es demostrar la potencialidad de la didáctica del objeto como herramienta para la mejora de la didáctica de la Historia. Seleccionada una fuente primaria objetual cualquiera del pasado, se ha procedido a analizarla desde diferentes perspectivas para determinar el grado de conocimiento que su análisis permite en referencia al espacio y tiempo con que se relaciona la fuente. Asimismo, se pretende conocer si dicho análisis objetual posibilita obtener conclusiones generales a partir de premisas particulares, fomentando de este modo el método inductivo de aprendizaje. El presente trabajo pretende contribuir a mejorar la calidad de la didáctica de la Historia en particular, así como de los procesos de enseñanza-aprendizaje de las Ciencias Sociales, en general.

\section{Palabras clave}

Didáctica del objeto, Enseñanza-aprendizaje de la Historia, Fuentes Primarias, Investigación Didáctica, Investigación en Ciencias Sociales.

\footnotetext{
Abstract

The purpose of this research is to demonstrate the potential of object didactics as a tool to improve the teaching and learning of History. Once having selected any objectual primary source from the past, we proceeded to analyse it from different perspectives in order to determine the degree of knowledge that its analysis allows, regarding both the space and time to which the source is related. Additionally, the research seeks to know whether that object analysis enables students to draw general conclusions from particular premises, thereby promoting the inductive learning method.

1 Para contactar con esta autora: Nayra Llonch Molina. Universitat de Lleida. nayra.llonch@didesp.udl.cat. 2 Para contactar con esta autora: Verónica Parisi Moreno. Universitat de Lleida. veronica.parisi@didesp.udl. cat.
} 
This paper aims to contribute to improving the quality of History teaching and learning in particular, as well as the teaching and learning of Social Sciences in general.

\section{Keywords}

Object-based learning, History Instruction, Primary Sources, Educational Research, Social Sciences Research.

\section{Introducción}

\subsection{Antecedentes}

Tradicionalmente en nuestro país, sin ser una característica exclusiva de éste, la enseñanza de la Historia en la escuela se ha basado en unos contenidos con una alta carga patriótica, cuya función era la de reforzar el sentimiento identitario y nacionalista, y una carga cultural de refuerzo a la autoestima como miembros de la nación y, en consecuencia, de tendencia etnocentrista (LópezFacal, 1995, 2003, 2008, 2010; Valls, 1991, 2007, 2012).

Es evidente que en la situación de globalización en que nos encontramos actualmente no es posible mantener el método tradicional de enseñanza de la Historia. Enseñar un relato cerrado y etnocentrista del pasado choca con la realidad polimórfica de nuestra sociedad. En consecuencia, es necesario promover otros métodos de enseñanza-aprendizaje acordes con la sociedad actual $\mathrm{y}$, por tanto, más eficaces para la transmisión del conocimiento del pasado. Teniendo en cuenta las exigencias sociales del momento presente, una nueva didáctica de la Historia debe promover actitudes y aptitudes tales como interpretar los hechos, contrastar las fuentes, plantearse preguntas pertinentes, elaborar hipótesis, detectar prejuicios, extrapolar situaciones y hacer deducciones, entre muchas otras (Prats y Santacana, 2011a, 2011b, 2011c, 2011d). Para promover estas actitudes existen métodos y estrategias muy diversas como, por ejemplo, la enseñanza mediante el patrimonio, mediante el juego o mediante el objeto, siendo este último el método en el que se basa la presente investigación.

\subsection{Didáctica del objeto}

Todos los objetos encierran un sinfín de significados y, por tanto, son portadores de conocimiento, pero para descubrirlos es necesario saber interrogarlos. La didáctica del objeto tiene como finalidad primera, precisamente, relacionar el objeto con los conocimientos que contiene, para hacerlos comprensibles, fijarlos en la memoria y para servir de elementos de anclaje de aprendizaje de nuevos conceptos (Durbin, Morris y Wilkinson, 1996; García, 1994; Santacana y Llonch, 2012).

Puesto que el objeto es algo concreto, dotado de materialidad y de características determinadas, este se puede observar, describir, tocar, oler, descomponer, clasificar, contextualizar, dibujar, puede sugerirnos cosas, puede ser comparado con otros objetos, etc. En definitiva, podemos iniciar un proceso de análisis que nos lleva a fijarnos en los detalles del objeto y nos permite conocer cómo es, su función y funcionamiento, a quién va destinado, su proceso de fabricación, las implicaciones económicas en dicho proceso, en la adquisición de las materias primas que lo componen y en su comercialización, etc. Para ello, el análisis debe ser sistemático y pasa por analizar el objeto desde el punto de vista morfológico, funcional, técnico, económico, sociológico, estético e histórico-cultural (Santacana y Llonch, 2012). Cada una de estas perspectivas de análisis responde a diversas preguntas, como puede verse a continuación (Tabla 1): 


\begin{tabular}{|c|c|}
\hline TIPO DE ANÁLISIS & INTERROGANTE \\
\hline Morfológico & ¿Cómo es? ¿Es simple o complejo? ¿Qué partes lo componen? \\
\hline Funcional & ¿Cómo funciona y para qué sirve? ¿A qué necesidad responde? \\
\hline Técnico & $\begin{array}{l}\text { ¿Cómo se ha fabricado? ¿Qué técnicas de fabricación se han } \\
\text { aplicado? ¿Qué nivel tecnológico tiene la sociedad que lo ha fabricado? }\end{array}$ \\
\hline Económico & ¿Cuál es el coste de su fabricación? \\
\hline Sociológico & ¿A quién va destinado? \\
\hline Estético & ¿Qué cánones estéticos sigue (si los sigue)? \\
\hline Histórico-cultural & ¿Por qué razones históricas y en qué contexto surgió? \\
\hline
\end{tabular}

Tabla 1. Perspectivas de análisis a las que los objetos pueden ser sometidos.

Fuente: elaboración propia.

Según lo anterior, cabe pensar que dando respuesta a estos interrogantes podremos entender por qué el objeto es como es, podremos relacionar las características del objeto con conceptos más generales, seguramente podremos llegar a conclusiones sobre el contexto histórico, social y cultural que lo vio nacer y en el que se utilizó, podremos detectar incluso cambios y continuidades vinculados al objeto $\mathrm{y}$, por tanto, también las necesidades que cubría en períodos determinados del pasado, etc. Por todo ello, creemos que construir estrategias de enseñanza-aprendizaje basadas en las premisas de la didáctica del objeto que, a su vez, tienen como punto de partida la potencialidad del análisis de aspectos concretos vinculados a dicho objeto y su contexto puede contribuir favorablemente a una didáctica de la Historia. En concreto, de una historia no basada en relatos cerrados más o menos manidos por los historiadores o los redactores de libros de texto, sino basada en las fuentes primarias del pasado (Prats y Santacana, 2011e) y, para el caso que nos ocupa, en los objetos del pasado.

\section{Marco teórico}

Bajo estas premisas, la presente investigación se realiza, como ya hemos dicho, a partir de fuentes primarias, es decir, aquellas que se produjeron de forma simultánea a los acontecimientos sobre los que nos informan. Estas fuentes pueden ser de naturaleza muy diversa (Prats y Santacana, 2011e), por ejemplo, fuentes materiales (objetos de todo tipo, monumentos, caminos, edificios...), fuentes escritas (periódicos, cartas, documentos legales...), fuentes iconográficas (ilustraciones, grabados...) y orales (relatos). De acuerdo con los autores, los objetos son fundamentales para el conocimiento acerca de una cultura (ya sea presente o pasada), puesto que en ellos se reflejan las formas de vida de los grupos humanos a quienes pertenecieron, su grado de desarrollo, sus actividades productivas, sus creencias, el contexto histórico en el que dicha cultura se sitúa, entre 
otros. En definitiva, las fuentes primarias son "la voz" de los hombres y mujeres del pasado. Y de entre los distintos tipos de fuentes, los objetos suelen ser más propicios para el aprendizaje, ya que son más atrayentes, puesto que, tal y como afirma Fontal (2012), todo ser humano sin excepción se relaciona con objetos; desde pequeños nos gusta tocarlos, olerlos; establecemos relaciones de posesión, etc., y, por tanto, aprendemos por experimentación a través de ellos. Por otro lado, la tangibilidad consustancial a la naturaleza material de los objetos los hace aptos desde el punto de vista didáctico desde edades muy tempranas, lo que no sucede con otras fuentes como las textuales, por ejemplo.

De acuerdo con García (1994), el valor de la información que proporcionan los objetos radica en que se trata de productos de la actividad humana; cada objeto es el resultado de una serie de actos intencionados que se imprimen en él de forma involuntaria. Este carácter involuntario de la información contenida en los objetos la hace a su vez una información objetiva y veraz. Por ello, el grado de fiabilidad de los objetos suele ser superior al de otras fuentes, puesto que habitualmente no fueron creados en calidad de testimonios del pasado para perpetuar una determinada memoria (MacGregor, 2012), como puede ser el caso, por ejemplo, de algunos monumentos, de muchos documentos escritos y de algunas fuentes gráficas. Así, a diferencia de, por ejemplo, un texto escrito, la información proporcionada por un objeto no "suele estar mediatizada por la interpretación o por la intencionalidad del autor" (García, 1994, p. 8). Como se ha dicho, los objetos deben ser interrogados, pues en ellos mismos está el fiel relato de la historia humana. Por ejemplo, del uso que se le daba a un objeto podemos deducir necesidades de esa época, o del proceso de fabricación podemos determinar el grado de desarrollo de la sociedad que lo produjo. En resumen, los objetos nos hablan de relaciones y necesidades del ser humano, de sus costumbres, tradiciones y creencias, de momentos históricos y de sociedades pasadas y actuales, entre muchas otras cosas. Por ejemplo, también señala esta autora que los objetos no hablan únicamente del grupo social que los utiliza, sino que además nos referencian a otros grupos sociales, como pueden ser aquellos que los diseñaron, comercializaron, desarrollaron, etc.

A nivel didáctico, "el uso de objetos fomenta la observación, la comparación, la deducción y otras habilidades" (Prats y Santacana, 2011e, p. 13). Durbin, Morris y Wilkinson (1996) afirman que el uso de fuentes objetuales contribuye a:

1. Aprender a valorar los objetos que nos rodean.

2. Aprender acerca de otros tiempos y culturas que comparan el presente con el pasado.

3. Acceder a información de acceso que no está escrita.

4. Estimular la motivación, el interés, la curiosidad, la creatividad, la investigación y el deseo de saber.

5. Permitir el aprendizaje activo, que proporciona una experiencia sensorial que nos ayuda en la construcción del pensamiento abstracto y a desarrollar la memoria.

6. Permitir una experiencia sensorial y táctil (pueden ser manejadas, medidas, observadas según su color, olor, textura, temperatura, etc.) que permite la observación en tres dimensiones.

7. Proporcionar el desarrollo de habilidades del lenguaje, a través de ejercicios de descripción que utilizan verbos, adjetivos, vocabulario diverso en cuanto a la forma (¿cómo es?), materiales (¿de qué está hecho?) y función (¿para qué sirve?).

8. Promover el cuestionamiento y plantear hipótesis.

9. Estimular el debate y la expresión oral, contribuir al desarrollo de la comprensión histórica y la adquisición de los conceptos históricos.

10. Desarrollar capacidades para la resolución de problemas.

11. Promover el desarrollo de determinados conceptos relacionados con el tiempo histórico: la cronología, el cambio, la continuidad y el progreso.

12. Promover la imaginación y la empatía histórica.

Para Santacana y Llonch (2012), los argumentos de carácter didáctico a favor de enseñar con los objetos son los siguientes:

1. Fijan la imagen del concepto: los objetos se pueden observar desde múltiples perspectivas 
y por ello permiten relacionar la imagen del objeto con el concepto que se quiere trabajar. Además, el objeto actúa como un elemento de referencia, permitiendo que el alumno se exprese mejor.

2. Atraen la atención del alumnado, pues se sienten atraídos por la posibilidad de trabajar mediante un soporte material. Más aún cuando este soporte no es fácilmente reconocible por los alumnos y permite la introducción de cuestiones enigmáticas acerca del objeto.

3. Permiten trabajar el método hipotético-deductivo que permite ir de lo general a lo particular.

4. También permiten trabajar el método inductivo, llegar de lo particular a lo general mediante inducciones.

5. El uso de objetos provoca situaciones empáticas y fomenta la imaginación.

6. Son inclusores de la mente, por tanto, son útiles para que los alumnos vinculen la nueva información a la ya existente en su estructura cognitiva.

7. Activan la mente de tal manera que al recurrir a un objeto se activa una cadena de conceptos relacionados al objeto, lo cual permite establecer una secuencia entre el objeto y el sistema conceptual.

8. Son un soporte real, lo cual es muy importante en el contexto en que nos encontramos, donde la virtualidad empieza a sobresalir por encima de la materialidad.

Finalmente, de acuerdo con Prats y Santacana (2011e), el alumnado debe aprender a ser competente en la lectura de las fuentes, cosa que le permitirá descubrir la información que el objeto lleva consigo y, para ello, debemos propiciar en los alumnos una mentalidad indagatoria y también crítica.

\section{Metodología}

En primer lugar, se procedió a la elección de la fuente primaria del pasado que sería materia de análisis. En este caso, la fuente escogida fue un artefacto originario de los pueblos del Pirineo leridano denominado vasulla (Figuras 1 y 2). Los motivos por los que se seleccionó este objeto son (1) que en el pasado fue un objeto cotidiano imprescindible en los hogares de dicha zona y, en tanto que cotidiano, ayuda a ponernos en la piel del otro, en su día a día, (2) que actualmente es un objeto en desuso y sobre él surgirán un sinfín de incógnitas, (3) que en la actualidad su uso y funciones la realizan múltiples objetos y sistemas muy distintos, lo cual permite percibir el paso del tiempo a través de los cambios, así como trazar una línea de tiempo.

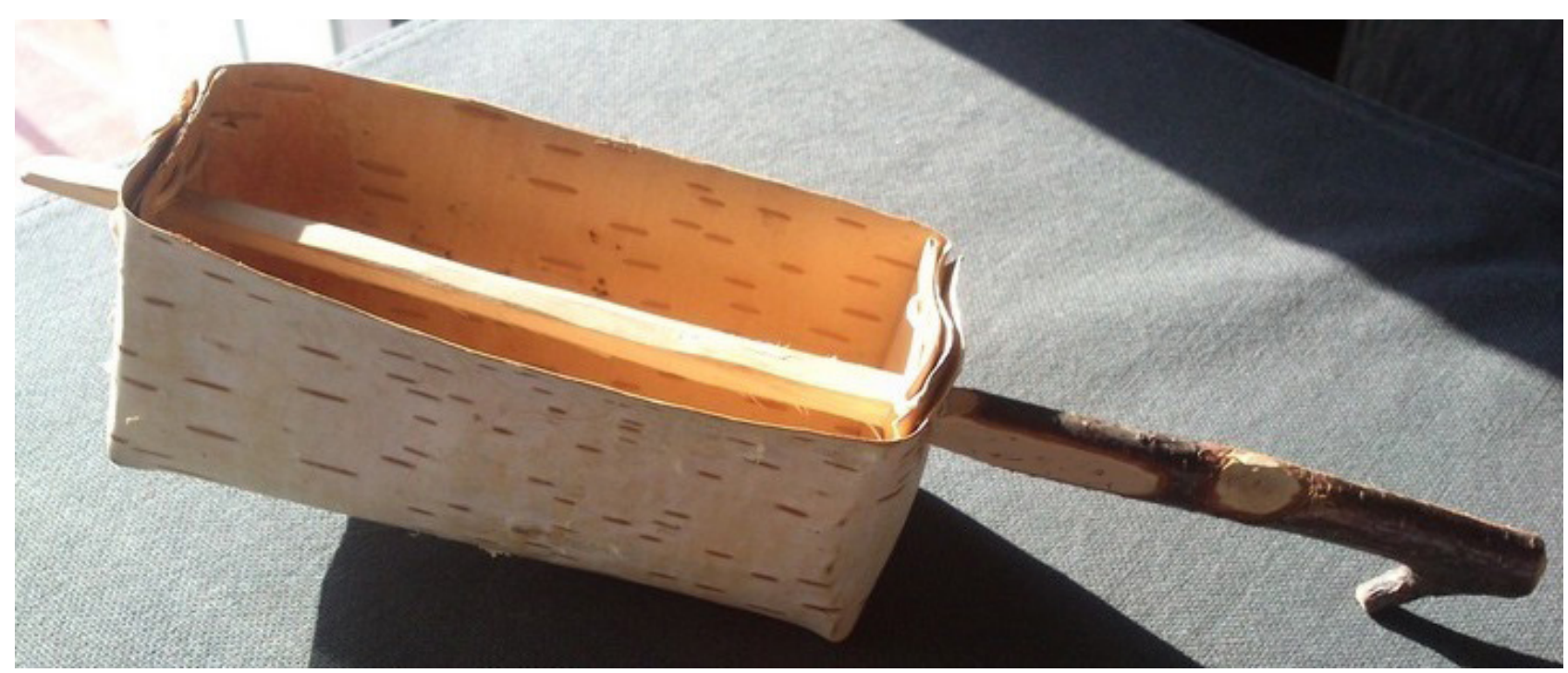

Figura 1: Vista en perspectiva de la vasulla. Fuente: Autoras. 


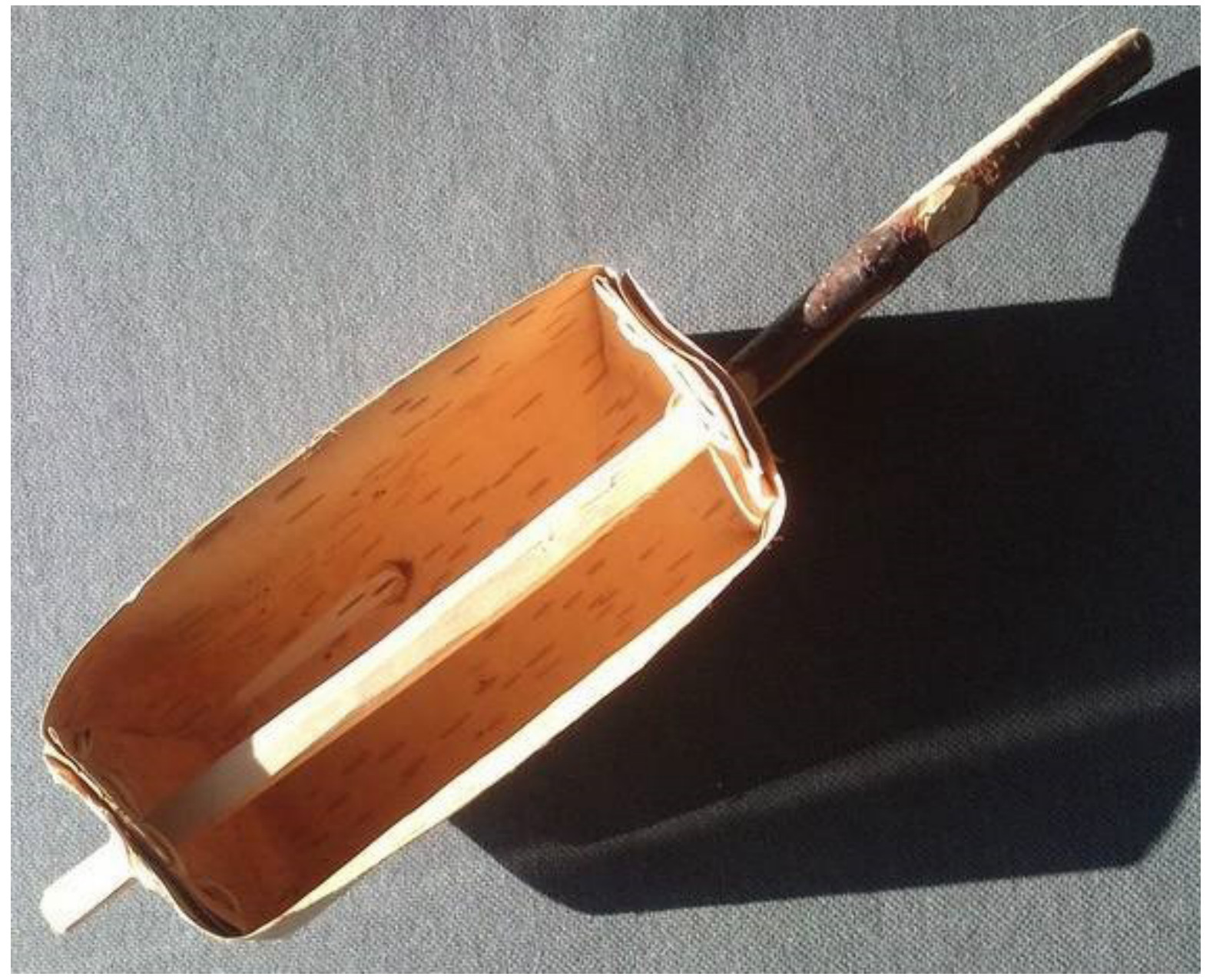

Figura 2: Vista cenital de la vasulla. Fuente: Autoras.

En segundo lugar, se seleccionó la pauta para el análisis del objeto. La guía de análisis escogida fue la elaborada por Santacana y Llonch (2012, p. 61) (Tabla 2). Los motivos de elección de esta guía son, en primer lugar, que puede ser aplicada a todo tipo de objetos, ya que se trata de un cuestionario genérico (si bien en algunos casos puede ser necesario realizar alguna adaptación según la naturaleza del objeto que estemos analizando). En segundo lugar, esta guía es adaptable según el nivel cognitivo de quien realiza el ejercicio, por lo que puede ser aplicada a todas las edades.

Identificación del objeto

- Nombre del objeto.

- Lugar donde se fabricó, donde se halló o donde está hoy.

- Autor o marca de producción, si existiera.

- Número de identificación, si lo tuviera

- Referencia bibliográfica, web, filmografía, etcétera. 
Análisis morfológico

- Descripción de la forma del objeto, intentando geometrizarla.

- Dimensiones del objeto que se analiza.

- Partes o piezas que lo componen, si ello es posible.

- Materiales que intervienen en la composición del objeto.

- Superficies, colores, etcétera.

- Conservación o estado actual (¿está roto o entero?, ¿tiene desperfectos?).

- Dibujo, croquis, esquema compositivo o fotografía.

Análisis funcional

- Qué utilidades puede tener el objeto, si es que se conocen.

- Describir cómo funciona o para qué podía utilizarse.

- Dilucidar, si es posible, la función de cada componente o parte.

- Relacionar la forma con la función.

- ¿Su forma facilita su función?

- Si utiliza energía para funcionar, decir cuál.

- ¿Presentaba riesgos su uso o funcionamiento?

Análisis técnico

- Describir, si es posible, las técnicas con las que se pudo fabricar o producir.

- Describir el proceso de producción, si es fácil deducirlo; realizar hipótesis.

- ¿Qué herramientas se pudieron haber utilizado?

- Características de los materiales utilizados en cada una de las partes.

- Ventajas o inconvenientes de los materiales usados.

Análisis económico

- Se trata de una producción propia, doméstica o foránea (manufacturada, industrial, etcétera).

- Estudiar cuánto pudo costar su fabricación (grado de especialización de los autores, dificultades técnicas, coste de los materiales, etcétera).

- Estudiar los costes de producción, es decir, los factores que implican importación de productos, transportes especiales, protección de rutas, intermediarios existentes en este comercio, etcétera. - ¿El objeto se podía producir con otros materiales a más bajos costes?

- ¿Quién o quiénes lo comercializaban, si fuera el caso?

Análisis sociológico

- Estudiar qué necesidades puede satisfacer el objeto.

- ¿A qué sectores puede ir dirigido?

- ¿Se trata de un elemento de uso general y masivo o de uso muy restringido?

- ¿Es un elemento muy común, accesible y barato para la mayoría?

- ¿Está específicamente pensado para alguno de los dos sexos o para algún tramo de edad?

Análisis estético o artístico

- Se trata de un objeto que guarda simetría o carece de ella.

- Propiedades de color, si fuera el caso.

- ¿La forma responde a la función?

- ¿Simple o complejo?

- ¿Responde a criterios estéticos actuales o no? 
Análisis histórico/cultural

- Cronología absoluta o relativa del objeto.

- En qué contexto surgió.

- ¿Fue o significó el objeto o este tipo de objetos un gran cambio con respecto a lo existente anteriormente?

- ¿El objeto representa una continuidad de algo anteriormente existente? ¿De qué?

- ¿Se puede relacionar con alguien o con algún movimiento o periodo?

- ¿Cómo evolucionó el objeto posteriormente? ¿Existe hoy algo parecido?

Tabla 2. Pauta para el análisis del objeto propuesta por Santacana y Llonch (2012, p. 61).

El análisis se llevó a cabo dentro de la asignatura Didáctica de las Ciencias Sociales Historia General de Europa, que se imparte en tercer curso del Grado de Educación Primaria en la Universidad de Lleida. El proceso tuvo una duración aproximada de un mes y se dividió en tres fases. La fase inicial consistió en una observación superficial del objeto y el planteamiento de las primeras cuestiones e hipótesis acerca del mismo. Posteriormente, en la fase de desarrollo, se procedió a dar respuesta a los apartados contenidos en la pauta de análisis del objeto. Para ello se recabó información procedente de diferentes fuentes primarias y secundarias (testimonios orales, bibliografía, otros objetos materiales relacionados con el que se estaba analizando, etc.). Para acabar, en la fase final del estudio se redactó el informe escrito que recogía todos los datos extraídos, las hipótesis realizadas y las deducciones e inducciones a las que se llegó mediante el análisis del objeto.

\section{Resultados}

La pieza concreta que se analizó fue fabricada en Escart, un municipio de la comarca del Pallars Sobirà, en la provincia de Lleida. Escart está situado a más de 1.200 metros de altura, tiene un clima mediterráneo de alta montaña y actualmente cuenta con 9 habitantes empadronados (Institut Estadístic de Catalunya, 2016).

En la identificación del objeto vemos que son varios los autores que han dejado constancia escrita sobre el mismo, desde el clásico Jacinto Verdaguer (1845-1902) en el poema El Canigó, un hito de la literatura catalana del período de la Renaixença, hasta autores contemporáneos como Pep Coll (1949). En estos textos vemos que no hay acuerdo sobre cuál es la forma original de la palabra. Según el autor se pueden leer las formas vasulla, beçulla, baçulla, bassulla, vassull, vessull o vasull, pero mayoritariamente se encuentra escrito en las dos primeras formas.

En el análisis morfológico del objeto ponemos de manifiesto sus dimensiones, analizamos su estructura, las diferentes texturas que presenta, colores, material de fabricación, etc. Por un extremo, la vasulla es cilíndrica y de tacto rugoso, mientras que el extremo opuesto resulta suave al tacto y tiene forma de prisma rectangular. Está compuesta por tres piezas: lo que parece un mango, lo que puede hacer las funciones de recipiente y un hilo que une el conjunto, manteniendo la estructura y la forma. Suponemos que el material del cual están fabricadas todas las piezas es algún tipo de árbol, pues el color del objeto es el color natural de la madera, por tanto, probablemente tampoco se haya hecho ningún tipo de tratamiento a la materia prima.

El análisis funcional descubre los principios sobre el funcionamiento del objeto, relacionando estructura y función. Por ejemplo, el recipiente podría servir para contener substancias líquidas ya que es impermeable, en ese caso, las funciones del mango podrían ser proporcionar comodidad al transportar el líquido y evitar quemaduras si se usaba la vasulla como recipiente para calentarlo. Puede ser por estos motivos que la rama que forma el mango no esté trabajada, así, al ser cilíndrica, es ergonómica y se adapta mejor a la forma de la mano. Por otra parte, vemos que se trata de un artefacto que no necesita ningún tipo de energía para funcionar. 


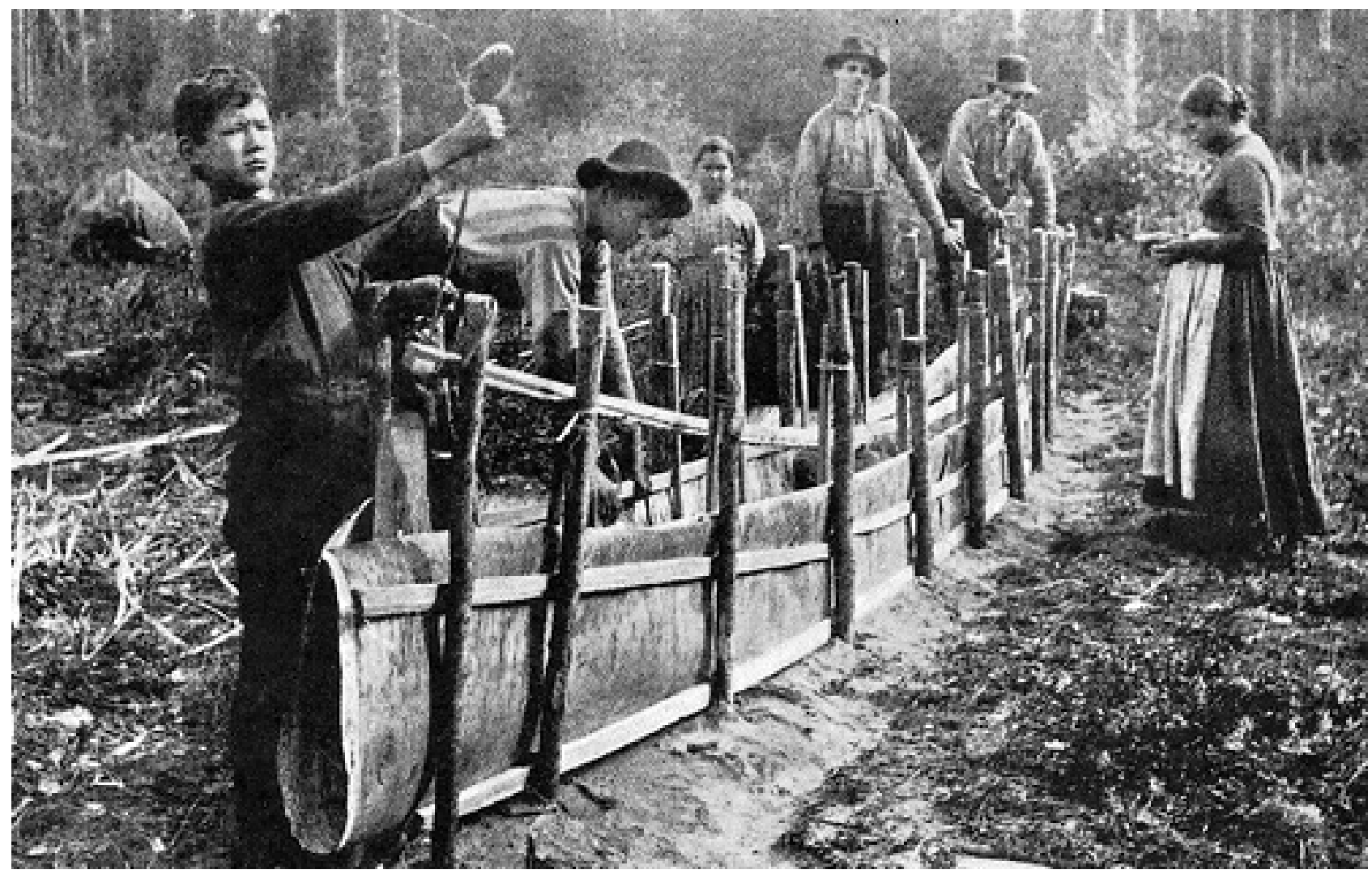

Figura 3: Los Menominee, en Wisconsin, son una tribu que aún existe, aunque actualmente la forman pocos individuos. Eran expertos fabricantes de canoas hechas de corteza de abedul. Fuente: Revista National

Geographic, noviembre de 1937.

El análisis técnico permite conocer y explicar cómo ha sido fabricado el objeto. Como sabemos, la vasulla que analizamos tiene su origen en una zona de alta montaña, donde generalmente el árbol más extendido es el abedul, ya que aquí halla condiciones óptimas para su crecimiento y expansión. El abedul es un material maleable y contiene una substancia llamada betulina que lo hace impermeable y lo conserva del paso del tiempo, cualidades que lo hacen idóneo para la fabricación de recipientes de todo tipo. Dentro de las ventajas del uso de este material, encontramos que el abedul crece con rapidez y en condiciones adversas de suelo y temperatura, como es el caso del Pallars Sobirà, donde el suelo es árido y las temperaturas a menudo llegan a extremos bajos. También encontramos que la madera de abedul es flexible; puede ser curvada o doblada por medio de calor, humedad o simplemente ejerciendo presión sobre ella. Otra ventaja es que el abedul, al tener dos cortezas, no muere cuando se le extrae la corteza exterior, además, esta corteza, a pesar de ser flexible, es muy fuerte y resistente. Por todo lo anterior, el uso del abedul para la fabricación de vasullas podría suponer una ventaja en cuanto al aprovechamiento de los recursos naturales del entorno.

Este apunte nos lleva a investigar si existen otros utensilios realizados a partir de la misma materia prima, lo cual nos lleva a descubrir que efectivamente hay otros lugares del mundo donde se realizan utensilios diversos con el mismo material. Por ejemplo, en Canadá se fabrican recipientes, de formas y tamaños variados, con o sin tapa, etc. Asimismo, localizamos en el norte de América una antigua tribu (Figura 3) conocida por haber sido sus miembros expertos fabricantes de canoas hechas de corteza de abedul, igual que el recipiente que forma la vasulla. Es más, descubrimos que en la región rusa de Novgorod se han encontrado rollos con inscripciones que datan de entre los siglos XI y XV, hechos también con corteza de abedul (Figura 4) y entre los cuales son especialmente 
remarcables unos que contienen inscripciones y dibujos realizados según los expertos por un niño de 6-7 años de edad llamado Onfim y que vivió en el siglo XIII (Figura 5). Estos documentos se han conservado hasta nuestros días gracias a que la corteza de abedul es una materia altamente resistente al paso del tiempo y la humedad.

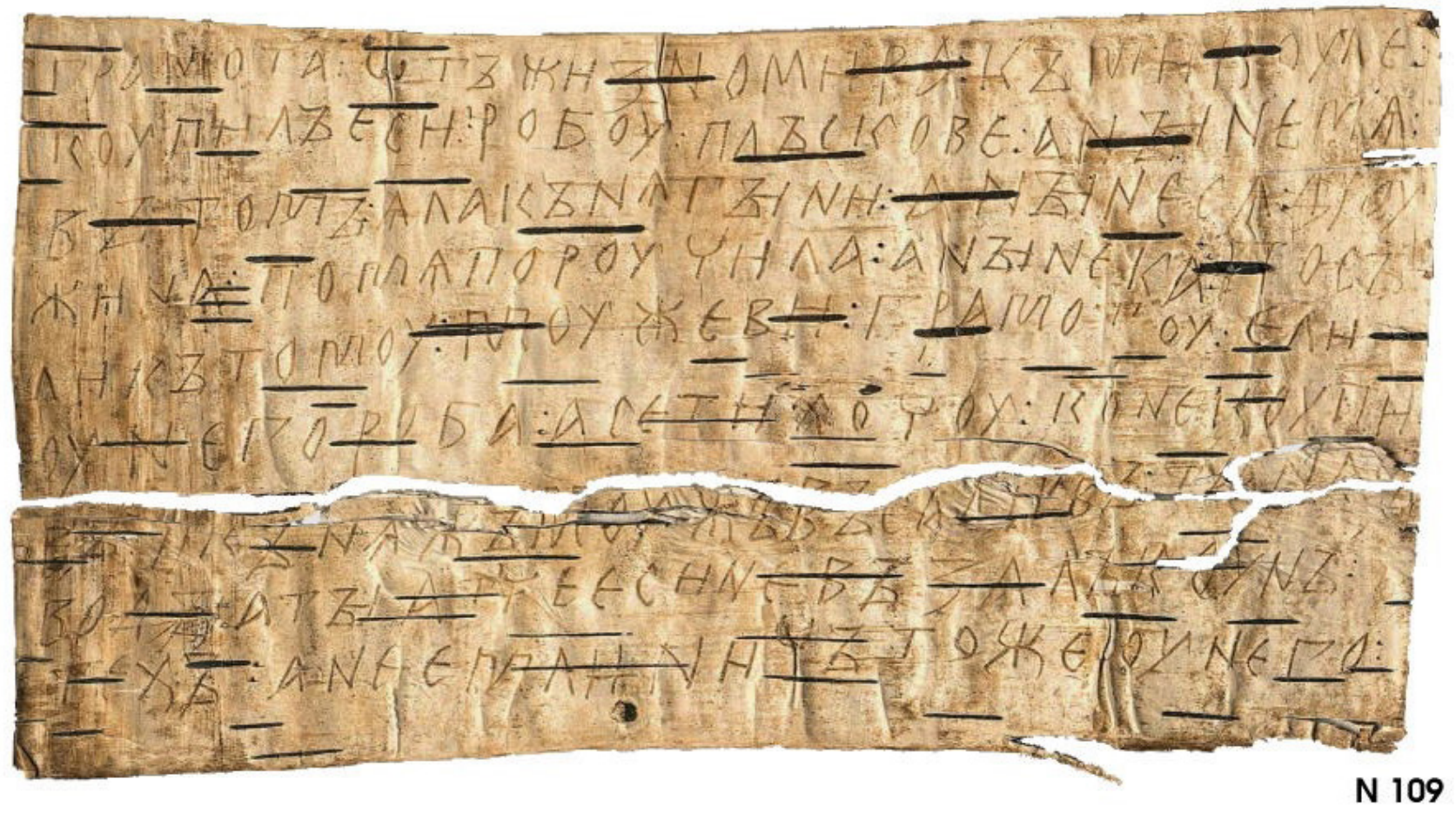

Figura 4: Carta de Zhiznomir a Micula, siglo XV. Fuente: Wikimedia Commons.

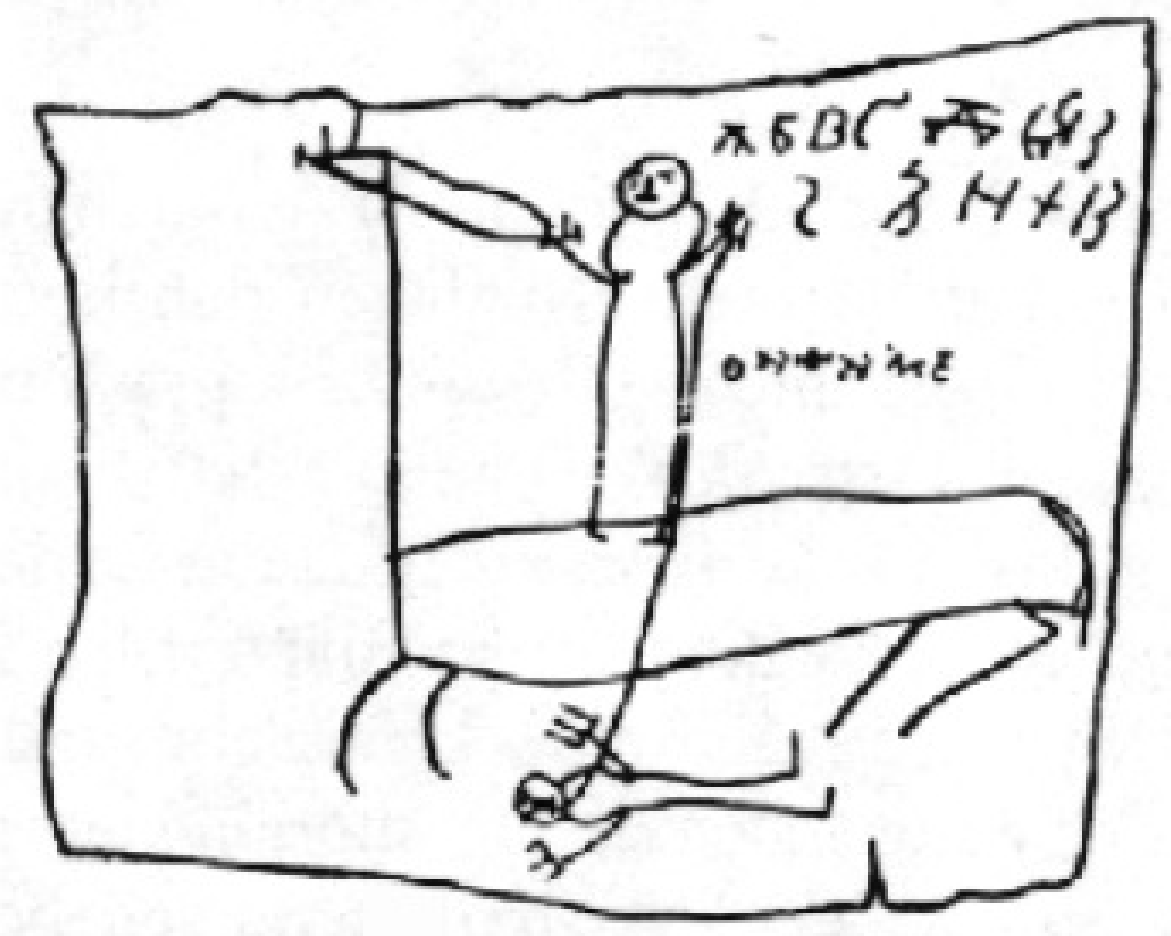

Figura 5: Dibujo de Onfim en corteza de abedul. Fuente: Attius, 2003. 


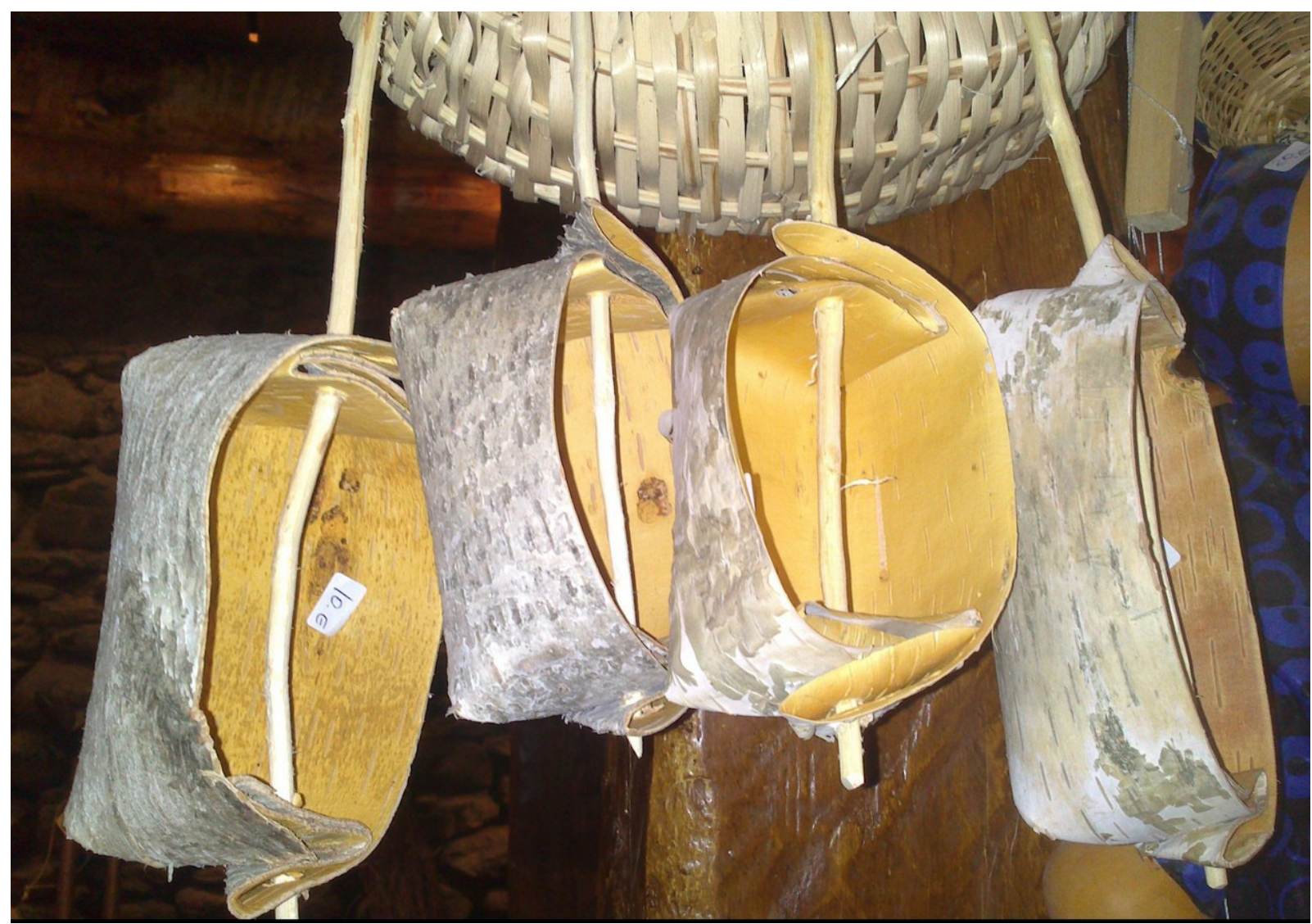

Figura 6: Vasullas en venta en el Ecomuseo de Esterri d'Àneu. Fuente: Autoras.

Mediante el análisis económico del objeto llegamos al concepto de desarrollo local. Al ser la materia prima el abedul que encontraban en el entorno, su coste de fabricación era cero. Así pues, no daba lugar a la comercialización del producto. En cambio, vemos que con la llegada del agua corriente a los hogares de la zona (durante la década de 1970), las vasullas dejaron de usarse como utensilio doméstico y empezaron a venderse como elemento decorativo, convirtiéndose de este modo en un elemento típico de la artesanía de la zona (Figura 6).

El análisis sociológico implica descubrir las necesidades que satisfacía la vasulla. Su fabricación y uso significaba un aprovechamiento de los recursos naturales del entorno para producir herramientas de consumo propio a coste muy reducido, es decir, responde a un contexto de economía de subsistencia. Así pues, la vasulla era un utensilio común, accesible, gratis y fácil de elaborar, en consecuencia, su uso era masivo y era un objeto muy utilizado. Otra característica de la economía de subsistencia de la zona era la explotación ganadera a nivel familiar. En este sentido, en todos los hogares había al menos un hombre que cuando conducía el ganado al pasto, para no tener que cargar tanto peso, llevaba consigo una vasulla en lugar de una cacerola. Por otro lado, vemos que la fabricación de vasullas era un entretenimiento para estos pastores en las largas jornadas de pasto. Sin embargo, aunque la fabricación fuese a cargo de los hombres, eran las mujeres quienes más utilizaban este utensilio, ya que eran ellas las que daban de beber a los animales, las que cocinaban e incluso las encargadas de ir a buscar provisiones de agua a la parte más alta del pueblo, para lo cual colocaban una vasulla dentro de cada cubo una vez lleno de agua limpia y, de este modo, la vasulla impedía que con el vaivén de bajada el agua se saliera de los cubos.

El punto siguiente, el del análisis estético, parte del estudio de la forma del objeto. Estamos frente a un objeto asimétrico, claramente manufacturado y de producción artesanal. No presenta decoraciones $y$, teniendo en cuenta su uso original como utensilio de cocina, las características 
de la vasulla no corresponden con los criterios estéticos actuales, ya que el menaje de cocina de hoy en día se fabrica principalmente de vidrio, cerámica, aluminio anodizado, acero inoxidable, teflón, esteatita, etc. A simple vista, parece que el objeto no ha recibido ningún tipo de tratamiento antes o después de su fabricación (barnices, aceites, etc.), su complejidad es mínima y no supone complemento alguno, sino que representa una prolongación del gesto humano de beber agua de la palma de la mano.

Finalmente, el análisis histórico del objeto permite contextualizarlo en un espacio y tiempo determinados, de características particulares. Aunque la cronología de la vasulla es relativa, pues se ha fabricado para uso doméstico desde tiempos inmemoriales, puede enmarcarse en el momento previo a la llegada de agua corriente a los hogares del Pirineo leridano, por ejemplo, en el período que va desde la Guerra Civil hasta la ratificación de la Constitución española, pasando por la posguerra y la dictadura. La vasulla muestra un ejemplo de cómo el conocimiento del medio y de sus recursos permite utilizarlos para dar respuesta a las necesidades de las personas en un momento histórico y un ámbito geográfico concretos. La vasulla surgió en un contexto de montaña, de escasos recursos económicos, pero muchos recursos naturales renovables, donde los hogares no tenían agua corriente, ni luz eléctrica, ni aseo; incluso los animales tenían una tarea dentro del funcionamiento de la vida cotidiana (por ejemplo, los gatos se encargaban de eliminar las ratas). No había conciencia medioambiental, pero se practicaban el reciclaje y la reutilización a gran escala, pues incluso lo que ya no se podía reutilizar podía ser útil para alimentar el fuego del hogar.

\section{Conclusiones}

En primer lugar, a lo largo del análisis se establecieron un gran número de conexiones entre el objeto y múltiples y variados conceptos. El análisis pautado de un objeto cualquiera nos informa en profundidad de un lugar y época con un determinado paisaje, tipo de clima, organización social, forma de consumo, sistema económico, incluso régimen político, etc. Así pues, no cabe duda del valor didáctico de los objetos a fin de construir imágenes, situaciones y contextos del pasado.

En segundo lugar, el análisis permitió extraer conclusiones generales a partir de casos particulares, del elemento concreto (el objeto) a lo abstracto (ideas, conceptos, procesos, formas de vida).

En tercer lugar, se encontraron relaciones entre el objeto analizado y otros objetos desarrollados por otras culturas. De este modo, la didáctica del objeto permite trasladarnos de lo local a lo global. El análisis también fue la vía para descubrir que las culturas, aunque habiten en partes opuestas del planeta, pueden tener las mismas necesidades y/o desarrollar soluciones similares a sus problemas, así como establecer diferentes maneras de aprovechar los mismos recursos con fines diferentes.

Consideramos además que es imprescindible que el análisis sea sistemático y se resuelvan todos los apartados, pues algunas perspectivas del análisis dependen directamente de otra u otras anteriores (como es el caso del análisis estético, que parte del análisis de la forma). De no seguir un proceso pautado de análisis, la cantidad de conocimiento e inducciones que podemos llegar a inferir no sería la misma.

Además de los conocimientos históricos y sociales adquiridos con el análisis del objeto, hemos comprobado que la didáctica del objeto potencia la capacidad de observación y descripción, activa el pensamiento, obliga a relacionar conocimientos, favorece la interpretación crítica, entre muchos otros procesos mentales. En definitiva, se trata de un proceso de enseñanza-aprendizaje que, por una parte, es activo ya que requiere de una participación integral y, por otra parte, es metacognitivo, pues el conocimiento adquirido es resultado del propio análisis elaborado. En este sentido, cabe mencionar también que el método de análisis del objeto conduce al descubrimiento, siendo este un proceso que puede darse a cualquier edad, ya que responde a la necesidad humana de aprender y entender.

Finalmente, y a la luz de los resultados de la investigación y conclusiones, podemos afirmar en este trabajo de investigación que la didáctica del objeto contribuye favorablemente a la mejora de 
la didáctica de la Historia, en tanto que facilita la comprensión de los hechos del pasado, así como promueve el ejercicio de los procesos de análisis e investigación propios de la disciplina histórica.

\section{Reflexiones finales}

La didáctica del objeto no forma parte de las actividades que se desarrollan en los centros educativos de nuestro país. Sin embargo, es importante rehacer el relato histórico a partir de las fuentes primarias del pasado, pues es el modo de salvaguardar la historia de influencias políticas y religiosas y desvincularla de nacionalismos y etnocentrismos. Los resultados positivos de la investigación avalan que es posible repensar la forma en que se transmite el conocimiento histórico y actuar en consecuencia.

Por otra parte, la didáctica del objeto resulta un método de enseñanza-aprendizaje inclusivo y motivador que responde a las necesidades e intereses del alumnado. No olvidemos que el objeto a analizar puede ser de tipología muy diversa y conviene, para aumentar su efecto didáctico, que el alumnado tenga su protagonismo a la hora de elegirlo.

Finalmente, este modelo de enseñanza-aprendizaje permite desarrollar diversas competencias tanto de obtención de conocimiento histórico como procedimentales, es decir, sirve tanto para el aprendizaje de la historia como para la adquisición de metodologías de enseñanza-aprendizaje de las ciencias sociales en general. En consecuencia, debería ser aplicado en las etapas de educación obligatoria, pero sobre todo es necesario que desde las facultades de educación se fomenten y pongan en práctica estos métodos en la formación inicial de maestros, pues al experimentar los futuros maestros y maestras esta forma de aprender la historia en primera persona se está aportando un modelo metodológico a seguir para cuando estos ejerzan su tarea profesional en las aulas.

\section{Bibliografía}

Attius, M. (2003). Hos gossen Onfim i Novgorod. Scriptum, 52, 126-127.

Durbin, G., Morris, S. y Wilkinson, S. (1996). A Teacher's Guide to Learning from Objects. London: English Heritage.

Fontal, O. (2012). Si som sensibles al patrimoni en tindrem cura. Si en tenim cura, l'altra gent en podrà gaudir. Guix, elements d'acció educativa, 381, 12-15.

García, A. (1994). Didáctica del museo. El descubrimiento de los objetos. Madrid: Ediciones de la Torre.

Institut Estadístic de Catalunya (2016). Recuperado el 28 de mayo de 2016, de http://www.idescat. $\mathrm{cat} /$ codis $/$ ? id $=50 \& \mathrm{n}=9 \& \mathrm{c}=259031$ \&lang=en

López-Facal, R. (1995). El nacionalismo español en los manuales de Historia. Educació $i$ història: Revista d'història de l'educació, 2, 119-128.

López-Facal, R. (2003). El concepto de nación en la enseñanza de la historia. Bulletin d'histoire contemporaine de l'Espagne, 32-36, 249-253.

López-Facal, R. (2008). Identificación nacional y enseñanza de la historia: 1970-2008. Historia de la educación: Revista interuniversitaria, 32-36, 171-193.

López-Facal, R. (2010). Nacionalismo y europeismo en los libros de texto: identificación e identidad nacional. Clío \& asociados: la historia enseñada, 14, 9-13.

MacGregor, N. (2012). La historia del mundo en 100 objetos. Barcelona: Debate.

Prats, J. y Santacana, J. (2011a). Por qué y para qué enseñar Historia. En J. Prats (coord.) Didáctica de la Geografía y la Historia (pp. 13-29). Barcelona: Graó.

Prats, J. y Santacana, J. (2011b). Los contenidos en la enseñanza de la Historia. En J. Prats (coord.) Didáctica de la Geografía y la Historia (pp. 31-49). Barcelona: Graó.

Prats, J. y Santacana, J. (2011c). Métodos para la enseñanza de la Historia. En J. Prats (coord.) Didáctica de la Geografía y la Historia (pp. 51-66). Barcelona: Graó. 
Prats, J. y Santacana, J. (2011d). Enseñar a pensar históricamente. En J. Prats (coord.) Didáctica de la Geografía y la Historia (pp. 67-87). Barcelona: Graó.

Prats, J. y Santacana, J. (2011e). Trabajar con fuentes materiales en la enseñanza de la Historia. En J. Prats (coord.), Geografía e Historia. Investigación, innovación y buenas prácticas (pp. 11-37). Barcelona: Graó.

Santacana, J. y Llonch, N. (2012). Manual de didáctica del objeto en el museo. Gijón: Ediciones Trea.

Valls, R. (1991). La exaltación patriótica como finalidad fundamental de la enseñanza de la historia en la educación obligatoria: una aproximación histórica. Didàctica de les Ciències Experimentals i Socials, 5, 33-47.

Valls, R. (2007). Historiografía escolar española: siglos XIX-XXI. Madrid: UNED.

Valls, R. (2012). La enseñanza española de la historia y su dimensión iberoamericana. Didáctica de las ciencias experimentales y sociales, 26, 121-143. 


\section{Panta Rei}

PANTA REI es una revista digital de investigación orientada a la Historia y otras ciencias afines. Su principal objetivo es la transmisión del conocimiento científico, dando una oportunidad también a los jóvenes investigadores que quieren abrirse camino en el estudio de las ciencias humanas y sociales. Se compone de estudios originales relacionados con la disciplina histórica así como su didáctica y difusión. Las diferentes secciones que componen la revista son: artículos de investigación, entrevistas a profesionales, recensiones de monografías de actualidad y crónicas de congresos o eventos científicos relevantes.

Todos los artículos publicados son objeto de un proceso de revisión a cargo de un mínimo de dos evaluadores, que se consideran expertos en el ámbito temático del artículo propuesto. Nuestro deseo es poder ofrecer unos contenidos rigurosos, de calidad y de interés.

EI CEPOAT (Centro de Estudios del Próximo Oriente y la Antigüedad Tardía de la Universidad de Murcia) es la institución encargada de la coordinación y gestión de la revista, desde donde anualmente se lanzará la convocatoria para aquellos que estén interesados en publicar sus trabajos, siempre relacionados con la Historia, Arqueología, Historia del Arte, Didáctica de las Ciencias Sociales, etc.

PANTA REI is a digital journal focused on History and other sciences related to it. Its main objective is the transmission of scientific knowledge by giving also an opportunity to young researchers who want to make their way in the study of human and social sciences. It is composed by original studies related to History, as well as its didactics and promotion. The different sections of this journal are: research articles, interviews to professionals, recensions on monographs about current issues and reports about congresses or relevant scientific events.

All the articles published are subject to a revision process carried out by a minimum of two reviewers who are considered to be experts in the field of the article proposed. Our wish is to offer rigorous contents with quality and being of interest to the reader.

CEPOAT (Centre of Studies of the Middle East and Late Antiquity of the University of Murcia) is the institution in charge of the coordination and management of this journal. This is the centre from where the call for papers will be launched for all the people interested in publishing their papers, always related to History, Archeology, Art History, Didactics of the Social Sciences, etc. 


\section{Normas de Publicación}

El autor se compromete a enviar trabajos originales, que no se encuentren publicados en otras revistas ni en otros idiomas. Así mismo, el mismo artículo no podrá ser presentado en otras revistas mientras dure el proceso de evaluación.

\section{Envío y presentación de originales}

Los artículos se enviarán exclusivamente a través del correo electrónico a la dirección pantarei@um.es. Los textos serán enviados en formato DOC y las imágenes en formato JPEG o TIFF, y con un tamaño mínimo de 2000 px. Éstas no aparecerán incorporadas en el texto, sino enviadas en archivo aparte y correctamente numeradas según su posición en el texto. Junto al trabajo, se rellenará y enviará un documento aparte en el que se especifiquen los datos del autor siguiendo el modelo disponible en la página Web de la revista.

Para la redacción de los trabajos se tendrá en cuenta el Manual de la American Psychological Association, en su sexta edición. La extensión máxima de los trabajos será de 30 páginas. La tipografía será Arial 11, con interlineado sencillo y sin espacio alguno entre párrafos. El texto deberá ir justificado a ambos márgenes y sin sangría en los primeros párrafos. Los márgenes serán de $2,50 \mathrm{~cm}$. En los casos en los que fuera necesario incorporar notas, éstas irán a pie de página, enumeradas consecutivamente, con tipografía Arial 10, interlineado sencillo y justificadas a ambos márgenes.

Una información más detallada se encuentra disponible en la página http://www.um.es/cepoat/ pantarei.

\section{Proceso de valoración y evaluación}

Una vez recibidos los trabajos, la Revista realizará una primera valoración. Si el trabajo enviado se ajusta a las normas de presentación propuestas, la temática es coincidente con la línea editorial de la revista y posee la calidad científica necesaria, será remitido al consejo asesor para una primera evaluación. Si no es así en este primer paso se puede rechazar directamente los documentos que incumplan claramente la línea editorial.

Será el Consejo Asesor quien indique a la revista la originalidad, relevancia, estructura, redacción, aparato bibliográfico, etc. del trabajo enviado y, para ello, se designará a dos revisores expertos externos que evaluarán cada uno de los trabajos, que pueden formar parte (o no) de este Consejo Asesor. La selección de los revisores se ajustará a la temática y características metodológicas del trabajo. El nombre y filiación de los autores serán eliminados del trabajo para su revisión, así como los revisores actuarán de manera anónima y confidencial.

Los revisores deberán rellenar un informe de evaluación que centrará su atención en aspectos tales como características formales, originalidad y novedad de los trabajos, relevancia de las propuestas y los resultados, calidad metodológica y validez científica.

Una vez terminado el proceso se decidirá la aceptación o no de los mismos y su publicación en el número que sea pertinente, así como las modificaciones susceptibles de ser realizadas para su final publicación. Dicha notificación se enviará únicamente por correo electrónico, en un plazo máximo de seis meses. 


\section{Publishing rules}

The author is committed to submit original papers not having been published in other reviews or in other languages. In this way, it is not allowed for the same paper to be presented in other reviews during the evaluation process.

\section{Submission and presentation of originals}

The articles will be exclusively submitted by email to pantarei@um.es. The texts will be submitted in DOC format and the images in JPEG or TIFF format, and with a minimum size of 2000 px. Images will not be integrated in the text but sent in another file and properly numbered according to their position in the text. Attached to the paper, a document will be filled out and sent where the author's data will be specified following the model available on the website.

The sixth edition of the Manual of the American Psychological Association will be taken into account for the writing of the papers. The length of the papers must not exceed 30 pages. Typography will be Arial 11 , with simple line spacing and no space between paragraphs. The text must be justified on both margins without indentation in the first paragraphs. Margins size will be $2.50 \mathrm{~cm}$. Where it could be necessary the incorporation of notes, they will be at the bottom of the page, consecutively numbered with typography Arial 10, simple line spacing and justified on both margins.

More detailed information is available on the website: http://www.um.es/cepoat/pantarei.

\section{Examination and assessment process}

The Journal will submit the papers to a first examination once received. If the paper follows the presentation guidelines, the subject agrees with the editorial line of this journal, and possess the scientific quality required, it will be sent to the advisory council for a first assessment. If not, the documents which clearly fail to complete the editorial line may be rejected straightaway in this first step.

The Advisory Council will indicate the originality, relevance, structure, writing, bibliography, etc. of the text to the journal; for this purpose, two outside experts will be designated to review the papers; these experts can be (or not) part of this Advisory Council. The selection of the experts will adjust to the subject and methodological characteristics of the paper. Name and affiliation of the author will be eliminated from the text for its review, in this way experts will act anonymously and confidentially.

The experts will fill out an assessment report which will focus on aspects such as formal characteristics, originality and novelty of the papers, relevance and results of the proposal, methodological quality and scientific validity.

Once the process is finished, the acceptance or not of the papers and its publication in the corresponding edition will be decided, as well as the modifications that may be done for its final publication. This notification will be sent by email within 6 months maximum. 


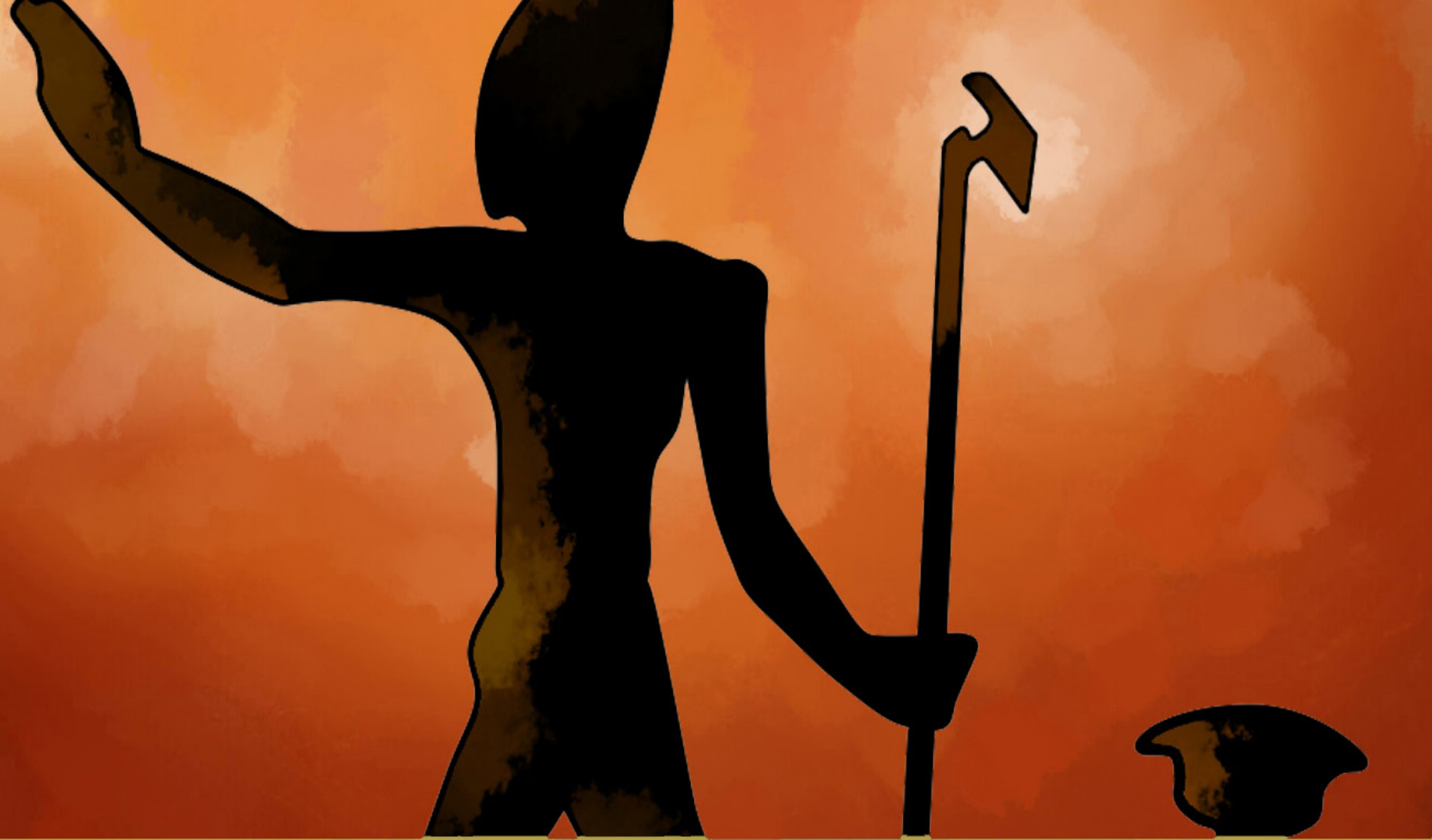

cepo t

UNIVERSIDAD DE MURCIA

centro de estudios del

próximo oriente y la

antigüedad tardía 